\title{
Genetic evaluation for bovine tuberculosis resistance in dairy cattle
}

\author{
G. Banos,${ }^{*} \dagger^{1}$ M. Winters, $\neq$ R. Mrode, ${ }^{*}$ A. P. Mitchell, $\S$ S. C. Bishop, $\dagger^{2}$ J. A. Woolliams, $\dagger$ and M. P. Coffey \\ *Scotland's Rural College, Midlothian EH25 9RG, United Kingdom \\ †Roslin Institute, University of Edinburgh, Midlothian EH25 9RG, United Kingdom \\ $\ddagger$ Agriculture and Horticulture Development Board (Dairy), Stoneleigh Park, Kenilworth, Warwickshire CV8 2TL, United Kingdom \\ §Animal and Plant Health Agency, Surrey KT15 3NB, United Kingdom
}

\begin{abstract}
Genetic evaluations for resistance to bovine tuberculosis (bTB) were calculated based on British national data including individual animal tuberculin skin test results, postmortem examination (presence of bTB lesions and bacteriological culture for Mycobacterium bovis), animal movement and location information, production history, and pedigree records. Holstein cows with identified sires in herds with bTB breakdowns (new herd incidents) occurring between the years 2000 and 2014 were considered. In the first instance, cows with a positive reaction to the skin test and a positive postmortem examination were defined as infected. Values of 0 and 1 were assigned to healthy and infected animal records, respectively. Data were analyzed with mixed models. Linear and logit function heritability estimates were 0.092 and 0.172 , respectively. In subsequent analyses, breakdowns were split into 2-mo intervals to better model time of exposure and infection in the contemporary group. Intervals with at least one infected individual were retained and multiple intervals within the same breakdown were included. Healthy animal records were assigned values of 0 , and infected records a value of 1 in the interval of infection and values reflecting a diminishing probability of infection in the preceding intervals. Heritability and repeatability estimates were 0.115 and 0.699 , respectively. Reliabilities and across time stability of the genetic evaluation were improved with the interval model. Subsequently, 2 more definitions of "infected" were analyzed with the interval model: (1) all positive skin test reactors regardless of postmortem examination, and (2) all positive skin test reactors plus nonreactors with positive postmortem examination. Estimated heritability was 0.085 and 0.089 , respectively; corresponding repeatabil-
\end{abstract}

Received August 21, 2016.

Accepted October 17, 2016.

${ }^{1}$ Corresponding author: Georgios.Banos@sruc.ac.uk

${ }^{2}$ Deceased. ity estimates were 0.701 and 0.697 . Genetic evaluation reliabilities and across time stability did not change. Correlations of genetic evaluations for bTB with other traits in the current breeding goal were mostly not different from zero. Correlation with the UK Profitable Lifetime Index was moderate, significant, and favorable. Results demonstrated the feasibility of a national genetic evaluation for bTB resistance. Selection for enhanced resistance will have a positive effect on profitability and no antagonistic effects on current breeding goal traits. Official genetic evaluations are now based on the interval model and the last bTB trait definition. Key words: genetic evaluation, bovine tuberculosis resistance

\section{INTRODUCTION}

Bovine tuberculosis (bTB) is a chronic bacterial disease of cattle caused by Mycobacterium bovis infection primarily involving the respiratory tract. The disease affects animal health and welfare, causing substantial financial strain to the dairy cattle sector worldwide through involuntary culling, animal movement restrictions, and the cost of control and eradication programs (Allen et al., 2010). Furthermore, bTB is considered a zoonotic disease with considerable public health implications in countries where it is not subject to mandatory eradication programs.

In Great Britain, the majority of bTB cases are recorded in south western England and Wales. A bTB control and eradication program has been in place in these areas since 1950 consisting of primarily routine and targeted surveillance of cattle herds, culling of positive animals, and movement restrictions on infected herds. Surveillance is based on the administration of the single intradermal comparative cervical tuberculin test (skin test) involving 2 separate injections of sterile purified mixtures of Mycobacterium avium and $M$. bovis antigens (tuberculins) in the deep layer of the skin of the neck, followed by examination of the skin for localized allergic reactions after $72 \mathrm{~h}$ (de la RuaDomenech et al., 2006). When reaction to the M. bovis 
tuberculin injection is deemed to be less than or equal to that to the $M$. avium tuberculin injection, then the skin test is considered negative (nonreactor). A positive skin test result (known as a reactor) is declared when the reaction to $M$. bovis tuberculin exceeds that to $M$. avium tuberculin by more than $4 \mathrm{~mm}$, according to the standard international interpretation (de la Rua-Domenech et al., 2006). In all other cases, the test is considered inconclusive and repeated $60 \mathrm{~d}$ later. If one or more animals in a herd react positively to the skin test, then a new bTB incident, also known as breakdown, is declared, prompting animal movement restrictions, suspension of the official bTB free (OTF) status of the herd, and systematic testing of all animals in the herd at $60-d$ intervals. Animals with a positive or 2 consecutive inconclusive skin tests are compulsorily slaughtered and examined at the abattoir for visible lesions of bTB in their organs. Tissue samples from a representative number of infected animals from each herd are submitted to the laboratory to isolate $M$. bovis in bacteriological culture. A positive postmortem examination result (presence of lesions, positive $M$. bovis culture, or both) signals a downgrading of the herd's OTF status from "suspended" to "withdrawn." The breakdown remains open and skin testing continues in the herd until 1 or 2 (depending on the postmortem results and location of the herd) consecutive negative tests at minimum intervals of $60 \mathrm{~d}$ are obtained on all remaining animals.

Implementation of bTB control and eradication programs incurs significant costs to taxpayers on an annual basis. During 2010-2011, these costs amounted to $£ 152$ million in Great Britain and $£ 23$ million in Northern Ireland (Abernethy et al., 2013). However, despite the investment and good control efforts, the incidence and prevalence of bTB cases in Great Britain constantly increased between the mid-1980s and 2012, although they have leveled off in more recent years. Even so, just over 4,800 new breakdowns were declared in cattle herds and more than 36,000 animals had to be slaughtered for bTB control purposes in 2015 (DEFRA, 2016). This has been partly attributed to a reservoir of endemic $M$. bovis infection in wildlife, especially badgers, in large parts of England and Wales. All these facts hinder progress toward achieving the DEFRA's goal for Great Britain to be OTF by the year 2038 .

The presence of genetic variation among individual animals in their immunological response to $M$. bovis exposure was documented by Pollock and Neill (2002). This genetic variation was subsequently quantified and moderate heritability estimates were reported in cattle (Bermingham et al., 2009; Brotherstone et al., 2010; Tsairidou et al., 2014). The amount of genetic variation and the level of estimated heritability render resistance to bTB amenable to improvement via genetic selection. Breeding for enhanced bTB resistance could complement existing control and eradication programs. However, relevant tools have not been widely available as no formal genetic evaluation systems have been put in place.

The objective of the present study was to assess the feasibility of a national genetic evaluation for bTB resistance in dairy cattle based on British population data. We combined data from various sources and developed automated data handling procedures suitable for a routine commercial process. We investigated different models and trait definitions.

\section{MATERIALS AND METHODS}

\section{Data}

Population surveillance data were made available from the Animal and Plant Health Agency (APHA) of the Department for Environment, Food and Rural Affairs (DEFRA). Data consisted of tuberculin skin test and postmortem examination records of dairy and beef cattle from Great Britain (predominantly England and Wales), spanning the period 1957-2014, although more than $90 \%$ of the recorded data were after 2000 . Skin tests had been applied to individual animals every 2 mo within a given breakdown (defined as the period of disease surveillance in a herd prompted by the first detection of an infected animal and ending with the lifting of herd movement restrictions). Animals were classified as nonreactors, inconclusive reactors, and reactors as described by de la Rua-Domenech et al. (2006).

Negative skin test results for individual animals (nonreactors) were not being systematically recorded in the APHA database before 2011. Therefore, the British Cattle Movement Service (BCMS) database was used to identify contemporaries of reactors and inconclusive reactors in the APHA database that were present in the same herd during each breakdown. All contemporaries found in the BCMS database that were not included in the APHA data were considered to be nonreactors. The combined APHA-BCMS data were merged with milk recording data to derive information about the date of calving and parity number of the animals. A final match with the national pedigree data set (including data from the official Herdbooks) maintained by the Edinburgh Genetic Evaluation Services on behalf of the Agriculture and Horticulture Development Board (Dairy), retrieved the identification of the sire of each cow. Figure 1 illustrates the combination of data from various sources. A total of 5,358,308 cow records were included in the initial project database. 


\section{Trait Definition}

The health status of each animal was defined as follows:

1. Infected: 3 definitions were examined:

a. Reactors to the skin test with positive postmortem examination results consisting of visible lesions of bTB, positive $M$. bovis culture, or both $(\mathbf{R}+\mathbf{P M})$; this conservative definition required that a positive skin test be confirmed postmortem and is consistent with the current formal APHA definition of a confirmed case as well as a previous study based on similar data (Brotherstone et al., 2010).

b. All reactors to the skin test regardless of postmortem examination results $(\mathbf{R})$; this definition was based on the very high specificity (ca. 99\%) and positive predicted value of the skin test (de la Rua-Domenech et al., 2006; Goodchild et al., 2015) implying a very small percentage of false positives (positive skin test reactors that were not actually diseased).

c. As in (b) plus nonreactors and inconclusive reactors to the skin test who had been subsequently slaughtered and had positive postmortem examination results $(\mathbf{R}+\mathbf{N P M})$; this definition aimed at capturing all information available that could be indicative of infection including possible false negative



Figure 1. Combination of data from different sources in the genetic evaluation for bovine tuberculosis (bTB) resistance; $\mathrm{APHA}=$ Animal and Plant Health Agency; BCMS = British Cattle Movement Service; EGENES = Edinburgh Genetic Evaluation Services. Color version available online. skin test reactors in the analysis (Allen et al., 2010).

2. Healthy: live nonreactors to the skin test or slaughtered nonreactors with negative postmortem examination results (i.e., absence of lesions and a negative $M$. bovis culture).

Based on the above, 3 trait definitions of the animal's bTB infection status were considered according to the 3 definitions of infected. The healthy animal definition was the same in all cases.

\section{Data Edits}

More than $90 \%$ of the records in the database were from breakdowns that started in the year 2000 or later. The latter data were also more complete in terms of postmortem examination results. Therefore, breakdowns that started before 2000 were removed from further analyses. This edit was consistent with a previous study conducted on similar data (Brotherstone et al., 2010). Additional edits kept only milking cows of the Holstein breed with an identified Holstein sire in breakdowns that were not shorter than 2 mo. A final edit required that breakdowns have at least 5 observations of which at least one pertained to an infected cow. According to the 3 trait definitions, data from 424,843, 642,995 , and 660,762 daughters of 15,211, 19,050, and 19,325 sires, respectively, were kept in the analysis.

\section{Genetic Evaluation}

In the first instance, the following animal model was used to analyze animal bTB infection status as defined above:

$$
\begin{aligned}
Y_{i j k m n} & =\mu+B_{i}+R_{j} \cdot M_{k}+L_{m} \\
& +b_{1} d u r+b_{2} a g e+b_{3} \text { phol }+A_{n}+e_{i j k m n},
\end{aligned}
$$

where $Y=\mathrm{bTB}$ infection status record of animal $n$ in breakdown $i(0 / 1), \mu=$ population mean, $B=$ fixed effect of the breakdown $i, R \cdot M=$ fixed effect of the interaction between calendar year $j$ and month $k$ of breakdown onset, $L=$ fixed effect of lactation number $m(m$ $=1$ for primiparous cows, 2 for multiparous cows), $d u r$ $=$ linear regression on duration of the breakdown $\left(b_{1}=\right.$ regression coefficient), age $=$ linear regression on age of animal at breakdown onset ( $b_{2}=$ regression coefficient $)$, phol $=$ linear regression on percentage of Holstein genes of the animal ( $b_{3}=$ regression coefficient), $A=$ random additive genetic effect of animal $n$ including pedigree $(6,398,839$ animals $)$, and $e=$ random residual. 
Although data were restricted to only Holstein cows, the percentage of Holstein (vs. British Friesian) genes was available in the national dairy pedigree and was included in the model, consistent with the national genetic evaluations for other traits (Edinburgh Genetic Evaluation Service, 2016).

In a separate analysis, a logit function was fitted to model 1 to account for the binary nature of the trait. In model 1, the entire breakdown irrespective of length represented a contemporary group (cohort of animals). Although the model adjusted for different breakdown duration, the time of exposure and actual infection could vary considerably within and across breakdowns, thereby affecting the true definition of the contemporary group and possibly affecting results. In an alternative design, breakdowns were split into equally sized (2 mo) intervals that would better capture the specific prevailing conditions and dynamics at a given time, and model exposure and infection consistently within and across breakdowns and herds. The interval duration of 2 mo was chosen in connection with bimonthly surveillance testing of herds during open breakdowns. As before, a breakdown interval was required to have at least one infected animal and a minimum size of 5 to be included in the analysis. Data from multiple intervals within the same breakdown were included, resulting in repeated records per individual cow. Specifically, animals defined as healthy in a given interval were assumed to have been healthy in all previous intervals within the same breakdown and were assigned repeated records of zero. An animal found to be infected in a given interval was assigned a record of 1 in this interval. In previous intervals within the same breakdown, this infected animal was assigned a value reflective of a diminishing probability of infection manifested as a record of $(0.40)^{\mathrm{n}}$, where $\mathrm{n}$ was the time distance from the interval of infection; for example, the infected animal record was 0.40 in the immediately previous interval, 0.16 in the interval before that, 0.064 in the third preceding interval, and so on. The probability of infection chosen $(0.40)$ is consistent with a sensitivity estimate of 0.60 of the skin test as diagnostic tool for bTB. Sensitivity reflects the proportion of negative skin test reactors (nonreactors) that were truly healthy; thus, the value of 0.40 represents the proportion of diseased nonreactors (false negatives). Reported sensitivity estimates of the tuberculin skin test range in literature from 0.51 to 0.81 (Downs et al., 2011; Álvarez et al., 2012; Karolemeas et al., 2012). Varying the assumed sensitivity and probability of infection between these values had only a trivial effect on the genetic evaluation results (data not shown).

The model of analysis under the interval design was revised as follows:

$$
\begin{aligned}
Y_{i j k l m n o} & =\mu+B_{i}+R_{j} \cdot M_{k}+L_{m}+D_{l}+b_{1} \text { age } \\
& +b_{2} \text { phol }+A_{n}+P E_{n}+e_{i j k l m n o}
\end{aligned}
$$

where $Y=$ bTB infection status record of animal $n$ in breakdown interval $i$ (repeated records), $B=$ fixed effect of the breakdown interval $i, R \cdot M=$ fixed effect of the interaction between calendar year $j$ and month $k$ of breakdown interval onset, $D=$ fixed effect of breakdown interval duration $l(l=1$ for a 2 -mo interval, 2 for a possibly shorter interval leading to the end of the breakdown), age $=$ linear regression on age of animal at breakdown interval onset $\left(b_{1}=\right.$ regression coefficient), $P E=$ random permanent environment effect associated with animal $n$. All other effects were as in model [1].

In all cases, variance component and parameter estimates were derived using the software ASReml (Gilmour et al., 2009) and genetic evaluations (estimation of breeding values) with the software MiX99 (Vuori et al., 2006). Reliability estimates of the genetic evaluations, reflecting the squared correlation between the estimated and true breeding values, were based on the approximation proposed by Jamrozik et al. (2000). Variance component estimation was based on a subset of data pertaining to sires with 20 to 500 daughters in the data. This edit resulted in about one-third of the data being used in variance component estimation, in each case.

Separate genetic evaluations were calculated after removing the last $2 \mathrm{yr}$ of data and repeating the analyses on the reduced data set. Results from the reduced and full data analyses were compared to test the stability of the genetic evaluation across time by emulating conditions of consecutive genetic evaluations with updated data. Additional model validation was conducted based on Interbull's method 3 for national genetic evaluations, which entails regression of current (full) on the previous (reduced) genetic evaluation and on a function of the number of new daughters per sire since the previous evaluation (Boichard et al., 1995). This function combines the number of new daughters by year of first calving with the total number of daughters in the current evaluation (Boichard et al., 1995).

\section{RESULTS AND DISCUSSION}

\section{Descriptive Statistics}

Table 1 summarizes the 3 data sets considered in the present study, depending on trait definition. In the breakdown design (model 1), each cow had a single record, whereas repeated records were included in the interval design (model 2). It should be noted that these proportions reflect only breakdowns with infected cases 
BANOS ET AL.

Table 1. Three data sets in the genetic evaluation according to bovine tuberculosis (bTB) trait definition ${ }^{1}$

\begin{tabular}{lccc}
\hline Item & $\mathrm{R}+\mathrm{PM}$ & $\mathrm{R}$ & $\mathrm{R}+\mathrm{NPM}$ \\
\hline No. of cows & 424,843 & 642,995 & 660,762 \\
No. of records $^{2}$ & $1,040,891$ & $2,170,322$ & $2,294,859$ \\
No. of sires of cows & 15,211 & 19,050 & 19,325 \\
No. of breakdowns & 4,365 & 8,158 & 8,397 \\
No. of breakdown intervals $^{2}$ & 7,585 & 18,079 & 18,822 \\
Proportion of infected cows $^{2}$ & 0.0357 & 0.0828 & 0.0829 \\
\hline
\end{tabular}

${ }^{1} \mathrm{R}+\mathrm{PM}$ : bTB infected $=$ skin test reactors with positive postmortem results; $\mathrm{R}$ : bTB infected $=$ all skin test reactors regardless of postmortem results; $\mathrm{R}+\mathrm{NPM}$ : $\mathrm{bTB}$ infected $=$ as $\mathrm{R}$ plus nonreactors and inconclusive reactors with positive postmortem results.

${ }^{2}$ Interval model only.

included in the present study and are not representative of the entire national herd.

As expected, the conservative definition of infection $(\mathrm{R}+\mathrm{PM}$, requiring a positive postmortem examination of skin test reactors) resulted in the lowest proportion of infected animals (3.57\%). A minimal difference was found between the other 2 data sets, which were mainly based on all skin test reactors regardless of postmortem results ( 8.28 vs. $8.29 \%$ ). The last data set also included nonreactors and inconclusive reactors that had been slaughtered and tested positively postmortem. However, very few such cases were present; in fact, of all infected cases in the third data set (R+NPM), 97.3\% were skin test reactors, $2.6 \%$ were inconclusive, and only $0.1 \%$ were nonreactors to the skin test.

\section{Breakdown vs. Interval Model}

Results from the breakdown design (model 1) and the interval design (model 2) were compared using the first trait definition $(\mathrm{R}+\mathrm{PM})$, where skin test reactors with positive postmortem results were considered to be infected. The heritability estimates were $0.093( \pm 0.009)$ and $0.115( \pm 0.014)$ for the 2 models, respectively. Heritability estimate after fitting a logit function to model 1 was $0.172( \pm 0.018)$, reflecting the genetic variation in the underlying liability scale. These estimates are in agreement with results of previous studies on British (Brotherstone et al., 2010) and Irish (Bermingham et al., 2009) bTB data considering the same trait definition. Presence of significant $(P<0.01)$ genetic variance signifies the amenability of the trait to improvement via selective breeding. Model 2 also yielded a repeatability estimate of $0.699( \pm 0.005)$ indicative of the definition of repeated records of the same cow within a breakdown in the present study.

Figure 2 shows the histogram of sire EBV by models 1 and 2. In accordance to industry preference, positive numbers were associated with higher resistance to bTB. Both models yielded normally distributed sire EBV. The average proportion of infected daughters among the top and bottom 20 bulls from the evaluation based on the breakdown model was 2 and $23 \%$, respectively. Corresponding proportions for the interval model were 2 and $24 \%$, respectively. Thus, the 2 models fared equally well at distinguishing sires whose offspring have a higher degree of resistance from those that are more susceptible.

Table 2 summarizes the reliability estimates of sire EBV obtained by the 2 models. Results are expressed as the cumulative percentage of sires falling within each reliability range. For example, 78 and $90 \%$ of the sires had EBV reliability greater than or equal to 0.30 based on the breakdown and interval model, respectively. Proportionally, more than twice the number of sires had EBV reliability of at least 0.50 based on the interval compared with the breakdown model, whereas this proportion was trebled for higher reliabilities $(\geq 0.60)$. The average sire EBV reliability was 0.40 and 0.54 for the breakdown and interval model, respectively. These results attest to the increased accuracy on the interval model, reflecting a more appropriate definition of the contemporary group and a larger amount of data in the genetic evaluation.

Figure 3 illustrates the relationship between sire EBV and proportion of infected daughters in the genetic evaluation. In both models, sire EBV were reflective of the infection rate among their daughters, with somewhat stronger correlations for the interval than the breakdown model $(-0.68$ vs. -0.64$)$. These correlations are expectedly negative as a higher EBV is indicative of increased resistance to bTB manifested by a lower infection rate.

Stability of genetic evaluations across time is illustrated in Figure 4. In both cases, sire EBV based on a reduced data set were very good predictors of EBV based on full data, the latter emulating a future genetic evaluation including new records. In this research case, new records were from an additional 2 full years of bTB surveillance, adding more than $30 \%$ of new data to the genetic evaluation. Official national genetic evaluations in the United Kingdom are calculated 3 times per year 
Table 2. Reliability of sire genetic evaluations ${ }^{1}$ based on the breakdown and interval models; cumulative percentage of sires per reliability range

\begin{tabular}{lcc}
\hline $\begin{array}{l}\text { Reliability } \\
\text { range }\end{array}$ & $\begin{array}{c}\text { Breakdown } \\
\text { model }\end{array}$ & $\begin{array}{c}\text { Interval } \\
\text { model }\end{array}$ \\
\hline$<0.10$ & 100 & 100 \\
$0.10-0.19$ & 94 & 97 \\
$0.20-0.29$ & 89 & 94 \\
$0.30-0.39$ & 78 & 90 \\
$0.40-0.49$ & 42 & 73 \\
$0.50-0.59$ & 22 & 53 \\
$0.60-0.69$ & 12 & 37 \\
$0.70-0.79$ & 7 & 25 \\
$0.80-0.90$ & 4 & 13 \\
$\geq 0.90$ & 2 & 6 \\
\hline
\end{tabular}

${ }^{1} \mathrm{bTB}$ infected $=$ skin test reactors with positive postmortem results.

meaning new data will be included more gradually leading to even higher correlations and stability between successive evaluation runs. High EBV correlations and stability across time are crucial for the acceptability of genetic evaluation results by the industry.

Validation with Interbull method 3 yielded a significantly greater than zero $(P<0.01)$ regression on the function of new daughters for the breakdown model but a nonsignificant one $(P=0.29)$ for the interval model. If a genetic evaluation is unbiased, this regression is expected to be zero (Boichard et al., 1995). Furthermore, Interbull require the regression to not exceed 0.02 genetic standard deviations to include a national genetic evaluation in their international comparisons (www.interbull.org). In the present study, the regression in question was 0.0338 and 0.0053 genetic standard deviations for the breakdown and the interval model, respectively, making the latter acceptable for national genetic evaluations.

The above results collectively demonstrate an overall superiority of the interval over the breakdown model in the analysis of bTB data. Therefore, further analyses were based on the former.

\section{Comparison of Trait Definitions}

The interval model was used to analyze data based on the other 2 trait definitions, where all skin test reactors $(\mathrm{R})$ and all skin test reactors plus nonreactors with

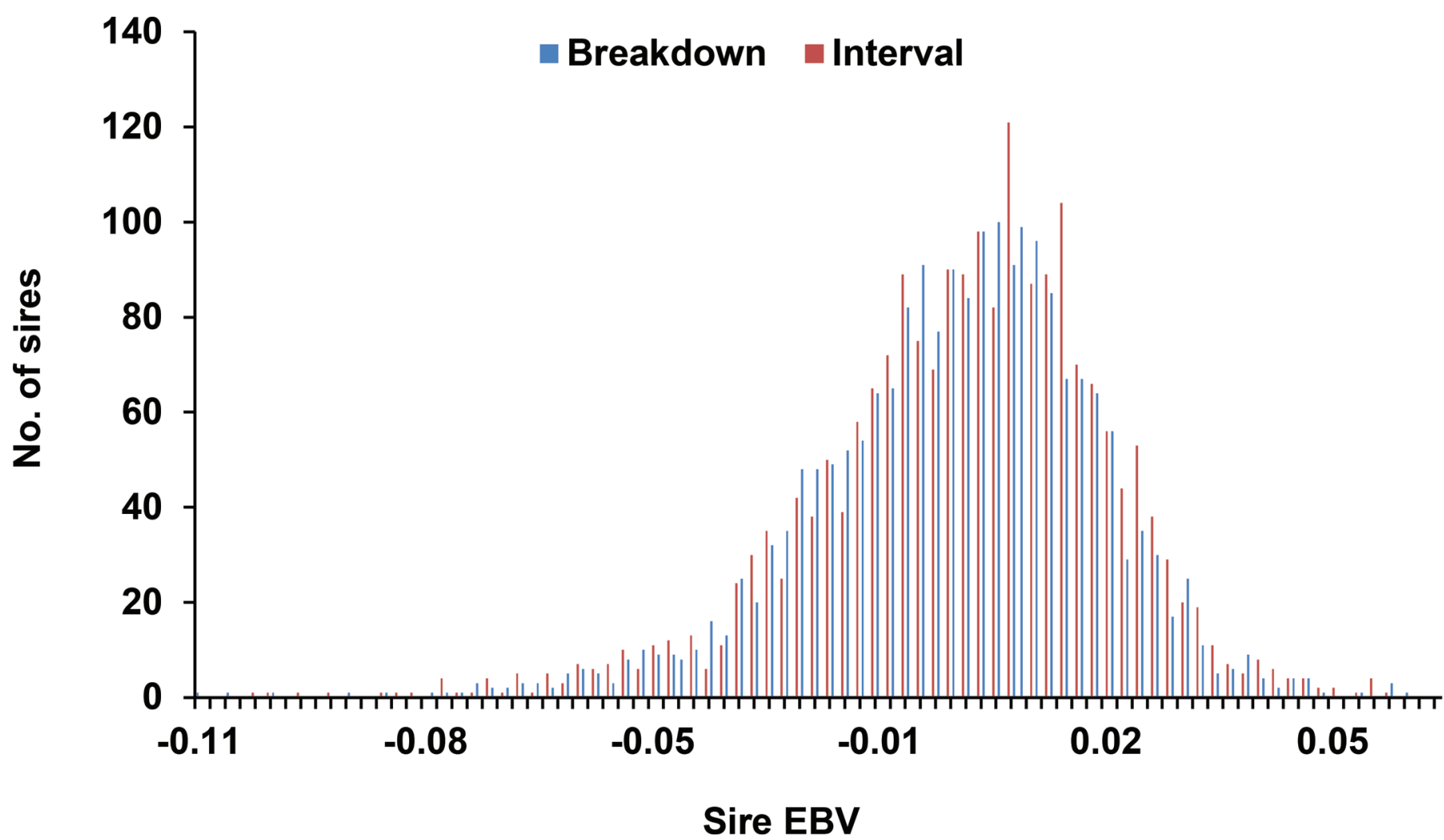

Figure 2. Histogram of sire EBV based on the breakdown and interval models; bovine tuberculosis (bTB) infected = skin test reactors with positive postmortem results. Color version available online. 

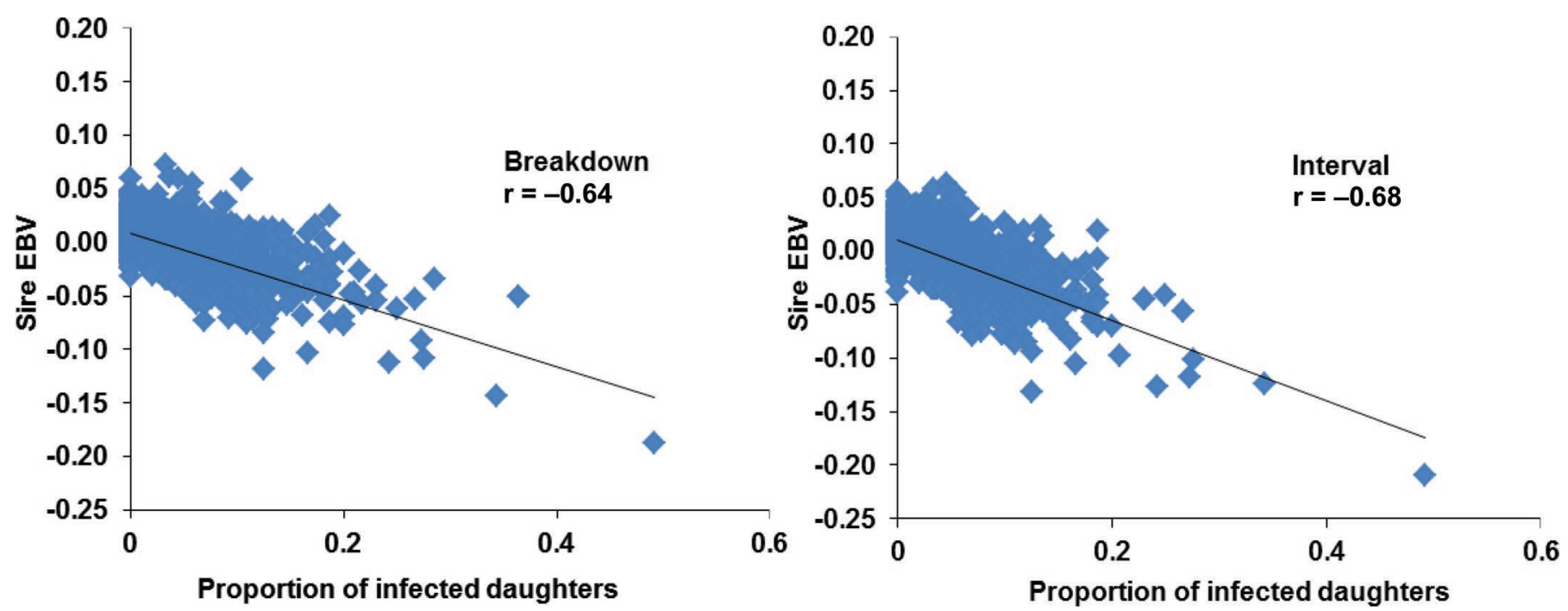

Figure 3. Sire EBV plotted against the proportion of infected daughters on which EBV were based, using the breakdown and interval models; bovine tuberculosis (bTB) infected = skin test reactors with positive postmortem results. Color version available online.

positive postmortem $(\mathrm{R}+\mathrm{NPM})$, respectively, were considered to be infected.

Table 3 summarizes the variance component and heritability estimates from the 3 interval model analyses. All estimates were statistically greater than zero $(P$ $<0.01$ ). Slightly higher heritability was estimated for the conservative definition of infected $(\mathrm{R}+\mathrm{PM})$, which can be attributed to the lower estimates for residual and permanent environmental variance (Table 3 ). The latter may be due to the definition of the trait, which, combined with the requirement to include breakdown intervals with at least one infected record, resulted in fewer records per cow compared with the more relaxed definitions ( $\mathrm{R}$ and $\mathrm{R}+\mathrm{NPM})$. In fact, the average number of records per cow increased from 2.45 in $\mathrm{R}+\mathrm{PM}$ to 3.38 and 3.47 for the other 2 definitions, respectively (Table 1). In all cases, genetic variance was of equal size and significant $(P<0.01)$, attesting to the amenability of all traits to genetic improvement via selection.

The distribution of sire EBV based on the $\mathrm{R}$ and $\mathrm{R}+\mathrm{NPM}$ trait definitions was similar to those in Figure 2 for the interval model $(\mathrm{R}+\mathrm{PM})$. Table 4 illustrates
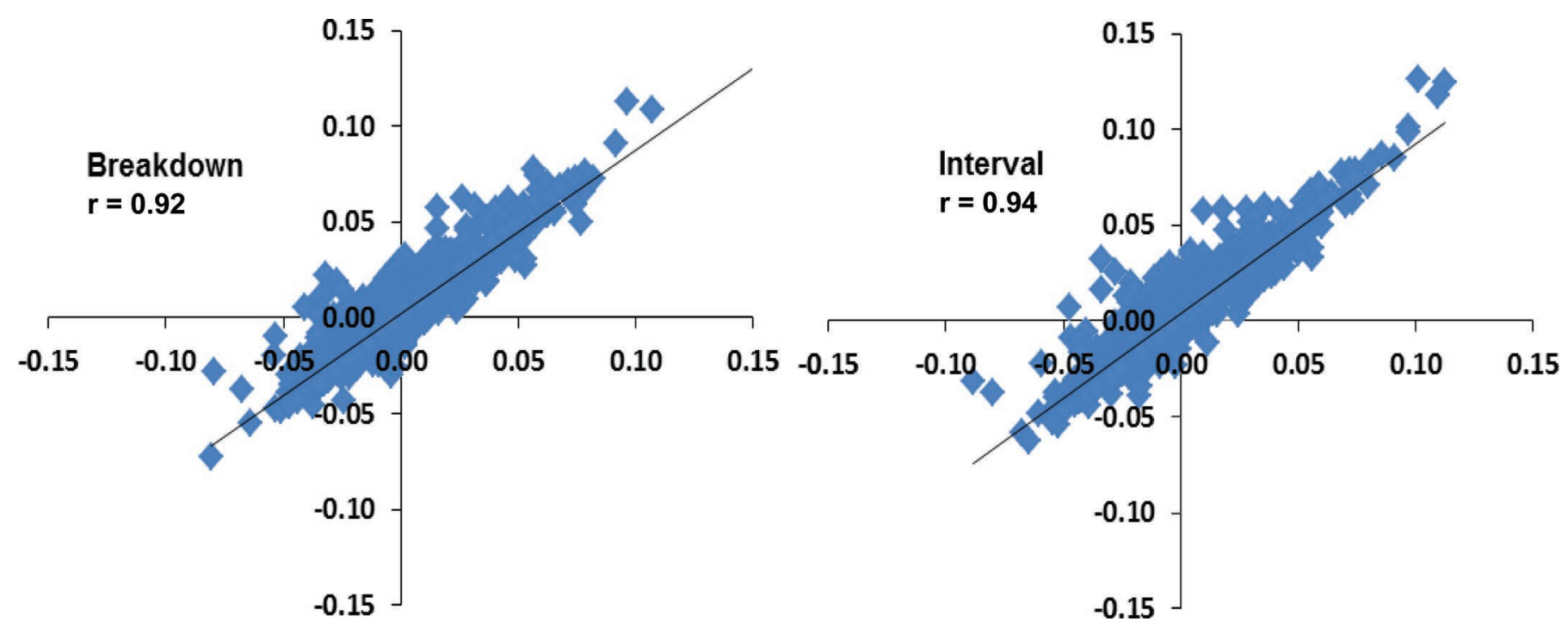

Figure 4. Sire genetic evaluations based on the full data set (vertical axis) plotted against genetic evaluations based on the reduced data set (minus last $2 \mathrm{yr}, 30 \%$ less), using the breakdown and interval models; $\mathrm{r}=$ correlation between genetic evaluations; bovine tuberculosis (bTB) infected $=$ skin test reactors with positive postmortem results. Color version available online. 
Table 3. Variance components and parameter estimates (Est.) and $\mathrm{SE}^{1}$ from the interval model analyses

\begin{tabular}{|c|c|c|c|c|c|c|}
\hline Item & \multicolumn{2}{|c|}{$\mathrm{R}+\mathrm{PM}$} & \multicolumn{2}{|c|}{$\mathrm{R}$} & \multicolumn{2}{|c|}{$\mathrm{R}+\mathrm{NPM}$} \\
\hline Permanent environment variance & 0.032 & 0.001 & 0.047 & 0.001 & 0.046 & 0.001 \\
\hline Residual variance & 0.016 & $<0.001$ & 0.023 & $<0.001$ & 0.023 & $<0.001$ \\
\hline Phenotypic variance & 0.055 & $<0.001$ & 0.076 & $<0.001$ & 0.076 & $<0.001$ \\
\hline Heritability & 0.115 & 0.014 & 0.085 & 0.007 & 0.089 & 0.007 \\
\hline
\end{tabular}

${ }^{1} \mathrm{R}+\mathrm{PM}$ : bovine tuberculosis (bTB) infected $=$ skin test reactors with positive postmortem results; R: bTB infected $=$ all skin test reactors regardless of postmortem results; $\mathrm{R}+\mathrm{NPM}$ : $\mathrm{bTB}$ infected $=$ as $\mathrm{R}$ plus nonreactors and inconclusive reactors with positive postmortem results.

differences between the top 20 and bottom 20 sires, by $\mathrm{EBV}$, in the 3 genetic evaluations. Sires with a minimum EBV reliability of 0.30 and daughters in at least 10 herds were considered in this table. The distinction between the best and worst sires was more pronounced in the $\mathrm{R}$ and $\mathrm{R}+\mathrm{NPM}$ cases compared with the conservative definition $(\mathrm{R}+\mathrm{PM})$. This can be attributed to the more relaxed definition in the last 2 cases, allowing more infected individuals to be included in the analysis. Enhanced capacity to distinguish sires by their genetic merit is expected to facilitate genetic progress.

Average reliability of sire EBV was $0.54,0.54$, and 0.55 for the 3 trait definitions $(\mathrm{R}+\mathrm{PM}, \mathrm{R}$, and $\mathrm{R}+\mathrm{NPM})$, respectively. The distribution of sires across ranges of EBV reliability was very similar to the interval model results shown in Table 2 for the conservative definition $(\mathrm{R}+\mathrm{PM})$. The advantage of the larger amount of data and increased progeny group size in the last 2 definitions (33.8 and 34.2 daughters per sire, respectively) compared with R+PM (27.9) was seemingly offset by the increased heritability of the latter (Table 3).

Product moment correlations between sire EBV based on the 3 trait definitions are shown in Table 5 . As expected, correlations were strongest between the last 2 definitions considering all skin test reactors $(\mathrm{R}$ and $\mathrm{R}+\mathrm{NPM}$ ). Weaker correlations with $\mathrm{R}+\mathrm{PM}$ can be primarily attributed to the number of diseased animals that reacted positively to the skin test and were culled without having had the time to develop and exhibit postmortem lesions.
The stability of genetic evaluations across time was tested for all trait definitions and results were very similar to those in Figure 2. Correlations between reduced and full model EBV were 0.94, 0.95, and 0.95 for $\mathrm{R}+\mathrm{PM}, \mathrm{R}$, and $\mathrm{R}+\mathrm{NPM}$, respectively. Validation with the Interbull method 3 yielded very similar results in $\mathrm{R}$ and $\mathrm{R}+\mathrm{NPM}$ analyses to those for $\mathrm{R}+\mathrm{PM}$ described above. In all cases, the genetic evaluations were shown to be unbiased as far as this method is concerned.

Correlations between sire EBV for bTB with the interval model and official EBV for other traits in the current national breeding goal are shown in Table 6. Sire EBV with a minimum reliability of 0.30 and daughters in minimum 10 herds $(2,039-2,996$ sires, depending on trait definition) were considered for this purpose. These results illustrate the generally weak and favorable correlation between genetic evaluations for bTB and other important traits. The strongest correlation estimates (0.15) was with the overall Profitable Lifetime Index $(£ \mathbf{P L I})$, which effectively combines all economically important traits in one single value (Agriculture and Horticulture Development Board, 2016). Significant ( $P$ $<0.05$ ) correlations were also observed with lifespan, which describes the functional longevity of a cow, reflecting the probability of being involuntarily culled after adjusting for milk yield. Relatively stronger correlations pertaining to $\mathrm{R}$ and $\mathrm{R}+\mathrm{NPM}$ can be attributed to losses of animals that react positively to the skin test and have to be culled, regardless of the outcomes of postmortem examination. These estimates indicate

Table 4. Differences between top 20 and bottom 20 sires in genetic evaluations based on 3 data sets ${ }^{1}$ and the interval model; sires with minimum reliability of 0.30 and daughters in at least 10 herds were considered

\begin{tabular}{lccc}
\hline Item & $\mathrm{R}+\mathrm{PM}$ & $\mathrm{R}$ & $\mathrm{R}+\mathrm{NPM}$ \\
\hline Difference in \% of infected daughters & 22 & 33 & 35 \\
Difference in EBV & 0.17 & 0.21 & 0.21 \\
\hline
\end{tabular}

${ }^{1} \mathrm{R}+\mathrm{PM}$ : bovine tuberculosis (bTB) infected = skin test reactors with positive postmortem results; $\mathrm{R}$ : bTB infected $=$ all skin test reactors regardless of postmortem results; $\mathrm{R}+\mathrm{NPM}$ : bTB infected $=$ as $\mathrm{R}$ plus nonreactors and inconclusive reactors with positive postmortem results. 
Table 5. Product-moment correlations between genetic evaluations (above diagonal) and number of common bulls (below diagonal) based on 3 data definitions ${ }^{1}$ and the interval model

\begin{tabular}{lccc}
\hline Item & $\mathrm{R}+\mathrm{PM}$ & $\mathrm{R}$ & $\mathrm{R}+\mathrm{NPM}$ \\
\hline $\mathrm{R}+\mathrm{PM}$ & & 0.62 & 0.64 \\
$\mathrm{R}$ & 14,998 & 19,050 & $>0.99$ \\
$\mathrm{R}+\mathrm{NPM}$ & 15,201 & 199 & \\
\hline
\end{tabular}

${ }^{1} \mathrm{R}+\mathrm{PM}$ : bovine tuberculosis (bTB) infected $=$ skin test reactors with positive postmortem results; $\mathrm{R}$ : $\mathrm{bTB}$ infected $=$ all skin test reactors regardless of postmortem results; $\mathrm{R}+\mathrm{NPM}$ : bTB infected $=$ as $\mathrm{R}$ plus nonreactors and inconclusive reactors with positive postmortem results.

that selection for increased resistance to bTB may have small favorable effects on $£$ PLI and cow longevity. In general, Table 6 suggests that no antagonistic effects on animal traits already in the breeding program should be expected from sire selection for enhanced bTB resistance. This is consistent with the UK £PLI placing over $65 \%$ of its emphasis on health traits.

The availability of bTB resistance genetic evaluations provides the industry with several options to add to the existing control measures. Farmers may choose to avoid particularly poor bulls when another bull of similar $£$ PLI is available. Breeding companies may make only desirable bulls available in high risk areas and may incorporate bTB in their bull dam choices where possible. These choices combined and made over time would be expected to lead to a general reduction in the infection rate in UK herds.

The bTB evaluations are now being used to create genomic breeding values. At the cow level, genomic breeding values would allow farmers to exclude young animals at an early age if they were predicted to be particularly susceptible to bTB. For example, if farmers removed the worst $5 \%$ of their animals each year before they had a chance to infect the remainder of the herd, the expectation would be that the overall level of herd infectivity would decrease over time and, therefore, the potential of each animal to infect another would be reduced. Similarly, the potential of a herd to pass infection to wild reservoirs would be reduced, thereby further decreasing the overall level of infectivity in the population. The genetic epidemiology of such a proposed policy warrants further study to determine an optimal strategy for the use of genetic evaluations in reducing overall bTB infection.

\section{CONCLUSIONS}

The feasibility of a genetic evaluation for enhanced bTB resistance using nationally available data was demonstrated in the present study. Results have shown that selective breeding can potentially make a positive
Table 6. Genetic evaluation correlations between bovine tuberculosis $(\mathrm{bTB})^{1}$ and other traits

\begin{tabular}{lrcc}
\hline Trait & $\mathrm{R}+\mathrm{PM}$ & $\mathrm{R}$ & $\mathrm{R}+\mathrm{NPM}$ \\
\hline Milk yield & 0.00 & 0.05 & 0.06 \\
Fat yield & -0.02 & $0.08^{*}$ & $0.08^{*}$ \\
Protein yield & 0.01 & $0.10^{*}$ & $0.10^{*}$ \\
Fat \% & -0.02 & 0.02 & 0.01 \\
Protein \% & 0.02 & $0.07^{*}$ & 0.06 \\
Milk SCC & -0.04 & -0.05 & -0.06 \\
Fertility index & 0.03 & 0.05 & 0.05 \\
Calving interval & 0.00 & -0.03 & -0.03 \\
Conception rate & 0.06 & 0.06 & 0.05 \\
Calving ease (direct) & 0.06 & $0.08^{*}$ & $0.08^{*}$ \\
Calving ease (maternal) & 0.04 & 0.06 & $0.07^{*}$ \\
Lifespan & 0.07 & $0.10^{*}$ & $0.11^{*}$ \\
Profitable lifetime index & 0.06 & $0.15^{*}$ & $0.15^{*}$ \\
\hline
\end{tabular}

${ }^{1} \mathrm{R}+\mathrm{PM}$ : bTB infected $=$ skin test reactors with positive postmortem results; $\mathrm{R}$ : $\mathrm{bTB}$ infected $=$ all skin test reactors regardless of postmortem results; $\mathrm{R}+\mathrm{NPM}$ : bTB infected $=$ as $\mathrm{R}$ plus nonreactors and inconclusive reactors with positive postmortem results.

${ }^{2}$ Combination of calving interval and nonreturn in $56 \mathrm{~d}$.

$* P<0.05$. Positive correlations are favorable except for milk SCC and calving interval.

contribution (when used alongside other interventions such as cattle movement restrictions and biosecurity improvements) to DEFRA's stated aim for Great Britain to be OTF by 2038. As of January 2016, the interval model has been applied in the official national genetic evaluation of Holstein sires considering all reactors to the skin test plus nonreactors and inconclusive reactors with positive postmortem results as infected individuals. Further work is planned to address bTB resistance in the other dairy breeds as well as beef cattle.

\section{ACKNOWLEDGMENTS}

Funding was provided by the Agriculture and Horticulture Development Board (Dairy), UK, and the Biotechnology and Biological Sciences Research Council, UK (BBSRC Reference: BB/L004054/1). Data were made available by the Animal and Plant Health Agency (Addlestone, UK), British Cattle Movement Service (Workington, UK), and Edinburgh Genetic Evaluation Services (Edinburgh, UK). Ian Archibald (Scotland's Rural College) compiled the datasets.

\section{REFERENCES}

Abernethy, D. A., P. Upton, I. M. Higgins, G. McGrath, A. V. Goodchild, S. J. Rolfe, J. M. Broughan, S. H. Downs, R. Clifton-Hadley, F. D. Menzies, R. de la Rua-Domenech, M. J. Blissitt, A. Duignan, and S. J. More. 2013. Bovine tuberculosis trends in the UK and the Republic of Ireland, 1995-2010. Vet. Rec. 172:312. https://doi. org/10.1136/vr.100969.

Agriculture and Horticulture Development Board. 2016. £PLI Profitable Lifetime Index. http://dairy.ahdb.org.uk/technicalinformation/breeding-genetics/\% C2\%A3pli/\#.Vx-XH3p8qRQ. 
Allen, A. R., G. Minozzi, E. J. Glass, R. A. Skuce, S. W. J. McDowell, J. A. Woolliams, and S. C. Bishop. 2010. Bovine tuberculosis: The genetic basis of host susceptibility. Proc. Royal Society B-Biological Sciences 277:2737-2745.

Álvarez, J., A. Perez, J. Bezos, S. Marqués, A. Graue, J. L. Saez, O. Mínguez, L. de Juan, and L. Domínguez. 2012. Evaluation of the sensitivity and specificity of bovine tuberculosis diagnostic tests in naturally infected cattle herds using a Bayesian approach. Vet. Microbiol. 155:38-43.

Bermingham, M. L., S. J. More, M. Good, A. R. Cromie, I. M. Higgins, S. Brotherstone, and D. P. Berry. 2009. Genetics of tuberculosis in Irish Holstein-Friesian dairy herds. J. Dairy Sci. 92:3447-3456.

Boichard, D., B. Bonaiti, A. Barbat, and S. Mattalia. 1995. Three methods to validate the estimation of genetic trend for dairy cattle. J. Dairy Sci. 78:431-437.

Brotherstone, S., I. M. S. White, M. Coffey, S. H. Downs, A. P. Mitchell, R. S. Clifton-Hadley, S. J. More, M. Good, and J. A. Woolliams. 2010. Evidence of genetic resistance of cattle to infection with Mycobacterium bovis. J. Dairy Sci. 93:1234-1242.

de la Rua-Domenech, R., A. V. Goodchild, H. M. Vordermeier, R. G. Hewinson, K. H. Christiansen, and R. S. Clifton-Hadley. 2006. Ante mortem diagnosis of tuberculosis in cattle: A review of the tuberculin tests, gamma-interferon assay and other ancillary diagnostic techniques. Res. Vet. Sci. 81:190-210.

Department for Environment, Food and Rural Affairs (DEFRA) 2016. Quarterly publication of the United Nations on the incidence and prevalence of tuberculosis (TB) in cattle in Great Britain - to end June 2016. Accessed Oct. 7, 2016. https://www.gov. uk/government/uploads/system/uploads/attachment_data/ file/529314/bovinetb-statsnotice-quarterly-15jun16.pdf.

Downs, S. H., J. Parry, J. Nunez-Garcia, D. A. Abernethy, J. M Broughan, A. R. Cameron, A. J. Cook, R. de la Rua-Domenech, A. V. Goodchild, M. Greiner, J. Gunn, S. J. More, S. Rhodes, S. Rolfe, M. Sharp, H. M. Upton, H. M. Vordermeier, E. Watson, W. Welsh, and A. O. Whelan. 2011. Meta-analysis of diagnostic test performance and modelling of testing strategies for control of bovine tuberculosis in GB. Pages 139-153 in Proceedings of the Society for Veterinary Epidemiology and Preventive Medicine, Leipzig, Germany. Society for Veterinary Epidemiology and Preventive Medicine, Edinburgh, UK.

Edinburgh Genetic Evaluation Service. 2016. EGENES. Accessed Oct. 7, 2016. http://www.sruc.ac.uk/info/120275/egenes.

Gilmour, A. R., B. J. Gogel, B. R. Cullis, and R. Thompson. 2009. ASReml User Guide Release 3.0. VSN International Ltd., Hemel Hempstead, UK. www.vsni.co.uk.

Goodchild, A. V., S. H. Downs, P. Upton, J. L. N. Wood, and R. de la Rua-Domenech. 2015. Specificity of the comparative skin test for bovine tuberculosis in Great Britain. Vet. Rec. 177:258. https:// doi.org/10.1136/vr.102961.

Jamrozik, J., L. R. Schaeffer, and G. B. Jansen. 2000. Approximate accuracies of prediction from random regression models. Livest. Prod. Sci. 66:85-92.

Karolemeas, K., R. R. de la Rua-Domenech, R. Cooper, A. V. Goodchild, R. S. Clifton-Hadley, A. J. K. Conlan, A. P. Mitchell, R. G. Hewinson, C. A. Donnelly, J. L. N. Wood, and T. J. McKinley. 2012. Estimation of the relative sensitivity of the comparative tuberculin skin test in tuberculous cattle herds subjected to depopulation. PLoS One 7:e43217. https://doi.org/10.1371/journal. pone.0043217.

Pollock, J. M., and S. D. Neill. 2002. Mycobacterium bovis infection and tuberculosis in cattle. Vet. J. 163:115-127.

Tsairidou, S., J. A. Woolliams, A. R. Allen, R. A. Skuce, S. H. McBride, D. M. Wright, M. L. Bermingham, R. Pong-Wong, O. Matika, S. W. J. McDowell, E. J. Glass and S. C. Bishop. 2014. Genomic prediction for tuberculosis resistance in dairy cattle. PLoS ONE 9:e96728.

Vuori, K., I. Strandén, M. Lidauer, and E. A. Mäntysaari. 2006. MiX99-Effective solver for large and complex linear mixed models. Pages 27-33 in Proc. 8th World Congress on Genetics Applied to Livestock Production, Belo Horizonte, Minas Gerais, Brazil, 13-18 August, 2006. Instituto Prociência, Brazil. 